\title{
PROPIEDADES PSICOMÉTRICAS DEL TEST DE CRIBADO DE DEMENCIAS PESOTEST EN MUESTRAS CLÍNICA Y NO CLÍNICA DE ADULTOS MAYORES
}

\author{
Martha Isabel Cantor Nieto, Bertha Lucía Avendaño Prieto* \\ Universidad Católica de Colombia
}

\author{
Recibido, julio 16/2015 \\ Concepto evaluación, marzo 7/2016 \\ Aceptado, abril 18/2016
}

\author{
Referencia: Cantor-Nieto, M. I. \& Avendaño-Prieto, B. L. (2016). \\ Propiedades psicométricas del test de cribado de demencias pe- \\ sotest en muestras clínica y no clínica de adultos mayores. Acta \\ Colombiana de Psicología, 19(2), 29-40. DOI: 10.14718/ \\ ACP.2016.19.2.3
}

Resumen

\begin{abstract}
El objetivo de este estudio instrumental fue determinar las propiedades psicométricas del test de cribado de demencias Pesotest en muestras clínica y no clínica de adultos mayores de Bogotá. Los participantes fueron 213 adultos mayores de 65 años. Los instrumentos utilizados fueron el Pesotest, el Mini Mental State Examination (MMSE), la Encuesta Sociodemográfica y la Escala de Depresión Geriátrica de Yesavage (GDS). La consistencia interna del Pesotest obtenida con el Alpha de Cronbach fue de 0.86. Se usó el MMSE para establecer la validez convergente y se encontró una correlación significativa de 0.596. Se hallaron diferencias estadísticamente significativas entre las muestras clínica y no clínica. El análisis factorial arrojó cuatro factores: mediante la curva ROC se encontró que la sensibilidad o proporción de personas diagnosticadas con demencia que dieron positivo fue de 0.83 ; la especificidad o proporción de personas sanas que según la prueba puntúan sin demencia fue de 0.78, y el punto de corte fue 18. El análisis con el modelo de Rasch evidenció que solo un ítem no se ajustó al modelo, y que el ítem con mayor dificultad era el que evaluaba memoria y cálculo, cuya alteración indica evolución a demencia. Con base en los resultados favorables del análisis psicométrico, adecuada consistencia interna, validez convergente, validez de criterio y validez de constructo, se recomienda utilizar el Pesotest en servicios de atención primaria.

Palabras Clave: Demencia, test, validez, confiabilidad.
\end{abstract}

\section{PSYCHOMETRIC PROPERTIES OF A SCREENING TEST FOR DEMENTIA PESOTEST IN CLINICAL AND NON-CLINICAL SAMPLES OF ELDERLY PEOPLE}

\begin{abstract}
The aim of this instrumental study was to establish the psychometric properties of the screening test for dementia Pesotest in clinical and non-clinical samples of elderly people. The participants of this study were 213 elderly people over 65 years old. The instruments used were the Pesotest, the Mini Mental State Examination (MMSE), the Sociodemographic Survey and the Geriatric Depression Scale Yesavage (GDS). A Cronbach's alpha of 0.86 for internal consistency of the Pesotest was found. The MMSE was used to establish convergent validity, finding a significant correlation of 0.596 . Statistically significant differences were also found between the clinical and nonclinical samples. The factor analysis yielded four factors and using the ROC curve it was found that the sensitivity, or the proportion of people diagnosed with dementia, was 0.83 ; and the specificity, or the proportion of healthy people that were diagnosed with dementia by the test, was 0.78 , with a cut-off point of 18. The analysis with the Rasch Model showed that only one item was not fit and that the most difficult item was the one which assessed calculation and memory, whose alteration indicates an evolution towards dementia. Based on the favorable results of the psychometric analysis, suitable internal consistency, convergent and construct validity and reliability, the use of the Pesotest in primary care services is recommended.

Key words: Dementia, test validity, statistical validity, test reliability.
\end{abstract}

\footnotetext{
* Universidad Católica de Colombia, Facultad de Psicología, Grupo ENLACE y Grupo GAEM, Av. Caracas No. 46 - 22, Bogotá, D.C., Colombia. (571) 3277300 Extensión 5050,micantor@ucatolica.edu.co, blavendano@ucatolica.edu.co
} 


\title{
PROPRIEDADES PSICOMÉTRICAS DO TESTE DE RASTREIO DE DEMÊNCIAS PESOTESTE EM AMOSTRAS CLÍNICA E NÃO CLÍNICA DE IDOSOS
}

\begin{abstract}
Resumo
O objetivo deste estudo instrumental foi determinar as propriedades psicométricas do Teste de Rastreio de Demências Pesoteste em amostras clínica e não clínica de idosos de Bogotá (Colômbia). Participaram do estudo 213 adultos maiores de 65 anos. Os instrumentos utilizados foram o Pesoteste, o Mini Mental State Examination (MMSE), a Pesquisa Sociodemográfica e a Escala de Depressão Geriátrica de Yesavage (GDS). A consistência interna do Pesoteste obtida com o Alpha de Cronbach foi de 0,86. Foi usado o MMSE para estabelecer a validade convergente e constatou-se uma correlação significativa de 0,596. Acharamse diferenças estatisticamente significativas entre as amostras clínicas e as não clínicas. A análise fatorial demonstrou quatro fatores: mediante a curva ROC, constatou-se que a sensibilidade ou a proporção de pessoas diagnosticadas com demência que deram positivo foi de 0,83 ; a especificidade ou a proporção de pessoas saudáveis que, segundo o teste pontuam sem demência, foi de 0,78 , e o ponto de corte foi 18. A análise com o modelo de Rasch evidenciou que somente um item não se ajustava ao modelo, e que o item com maior dificuldade era o que avaliava memória e cálculo, cuja alteração indica evolução a demência. Com base nos resultados favoráveis da análise psicométrica, na adequada consistência interna, na validade convergente, na validade de critério e na validade de construto, recomenda-se utilizar o Pesoteste em serviços de atenção primária.

Palavras-chave: demência, teste, validade, confiabilidade.
\end{abstract}

De acuerdo con las proyecciones del Departamento Administrativo Nacional de Estadística (DANE), en Colombia hay actualmente 4.628 .394 personas mayores de 60 años, cifra que equivale al $10 \%$ del total de la población. Para el año 2020, se estima que habrá alrededor de 6.500 .000 personas mayores, lo cual representa un crecimiento de 39,2\% respecto al 2011 (Rincón, 2013). Esta etapa de desarrollo está asociada con la presencia de diferentes problemáticas, entre estas el deterioro cognitivo y algún tipo de demencia. En Colombia algunos estudios sobre prevalencia de demencia en personas mayores de 50 años registraron cifras entre 1,3 y 5,4\% (Díaz, Ruano, Chacón, \& Vera, 2006; Pradilla, \& Vesga, 2002; Pradilla, Vesga, \& Bautista, 2000; Pradilla, Rosselli, \& Bautista, 1998), mientras que un estudio en mayores de 65 años en la ciudad de Neiva (Huila) estableció una prevalencia de 23\% (Gooding, Amaya, Parra, \& Ríos, 2006).

Las anteriores cifras evidencian que el psicólogo clínico y demás profesionales del área de la salud en atención primaria deben realizar el proceso de cribado, es decir, aplicar instrumentos que permitan detectar y seleccionar de manera breve y sencilla aquellos casos de quienes se sospecha tienen mayor probabilidad de presentar demencia, con el objetivo de remitirlos para estudio diagnóstico completo (Arango, Fernández \& Ardila, 2003; Carnero, 2005). Los tests de cribado deben cumplir, tanto con los requisitos de aplicabilidad (brevedad, facilidad, simplicidad, economía, aceptabilidad, equidad, adaptabilidad y flexibilidad) (Carnero, 2005), como con los requerimientos psicométricos de confiabilidad y validez a que son sometidas las pruebas psicológicas (Kerlinger, \& Lee, 2002; McIntire, \& Miller, 2000; Nunnally, \& Bernstein, 1995).
Los tests de cribado en idioma español presentan limitaciones relacionadas con tiempo de aplicación (Meulen et al., 2004), nivel educativo (Ostroskyj- Solis, López-Arango, \& Ardila, 1999) y población analfabeta (Rosselli et al., 2000; Peña-Casanova, Gramunt, \& Gich, 2004). En Colombia, el Mini Mental State Examination (MMSE), por su amplio uso, es la prueba que mejor permite el diálogo disciplinar e interdisciplinar. Es el único instrumento que cuenta con valores normativos estratificados por edad para la población colombiana. Por esta razón, se continuará utilizando mientras se carezca de validaciones de un test de cribado que presente también puntuaciones de corte establecidas de manera empírica (Arango et al., 2003). De los adultos mayores en el país, alrededor del $20 \%$ no tiene educación, $58 \%$ ha cursado solo la primaria, $17 \%$ tiene secundaria y $5 \%$ tiene educación superior (Profamilia, 2010). Por lo tanto, es importante contar con un test de cribado de demencia que pueda ser aplicado a personas analfabetas y no esté influenciado por el nivel educativo, como el test de cribado Eurotest (Carnero, \& Montoro, 2004).

Avendaño y Avendaño (2009) adaptaron este test al contexto colombiano con el nombre Pesotest. Lo aplicaron a 73 adultos mayores de 40 años, el $26 \%$ de ellos con diagnóstico de demencia y el $74 \%$ restante sin diagnóstico clínico. Los análisis de las características psicométricas mostraron consistencia interna, validez de contenido, validez convergente y validez de constructo. Se hallaron diferencias entre las muestras clínica y no clínica; el análisis factorial arrojó tres factores, la sensibilidad fue de 0.92 y la especificidad de 0.84 , para un punto de corte de 18 ; estos indicadores psicométricos coinciden con los estudios realizados por el autor del Eurotest. 
A partir de las siguientes consideraciones señaladas: aumento de población de adulto mayor, incremento de la prevalencia de demencias, ventajas de los tests de cribado, limitaciones de tiempo para la aplicación de pruebas en los servicios de salud, analfabetismo en Colombia, y de los resultados obtenidos por Avendaño y Avendaño (2009), se consideró pertinente realizar el estudio con una muestra más amplia y con adultos mayores de 65 años, dado que la prevalencia en este rango de edad es mayor. En consecuencia, el objetivo general de la investigación fue determinar las propiedades psicométricas (consistencia interna y validez) del test de cribado de demencias Pesotest en muestras clínica y no clínica de adultos mayores.

\section{MÉTODO}

\section{Tipo de investigación}

Este es un estudio instrumental dirigido al análisis de las propiedades psicométricas de una prueba (Montero, \& León, 2007) para establecer la presencia/ausencia de demencia.

\section{Participantes}

La muestra estuvo constituida por 213 adultos mayores de 65 años, 116 mujeres $(54,5 \%)$ y 97 hombres $(45,5 \%)$, procedentes de 14 departamentos de Colombia. Cundinamarca y Boyacá tuvieron la mayor representación con 96 $(45,1 \%)$ y $54(25,4 \%)$ personas, respectivamente. Del total de la muestra, 179 personas (84\%) sabían leer y escribir, y los restantes $34(16 \%)$ eran analfabetas; en el primer grupo, 112 personas $(52,6 \%)$ habían cursado primaria, $30(14 \%)$ secundaria, 9 (4\%) estudios técnicos, 9 (4\%) pregrado y 6 (3\%) posgrado; el 47 (22\%) no tenía ninguna educación formal.

Se contó con participantes de todos los estratos, con mayor concentración en el estrato $1(53,52 \%)$, seguidos del estrato $2(18,7 \%)$ y $3(15,96 \%)$.

En cuanto a la actividad laboral, $193(90,6 \%)$ no la realizaban, y $20(9,4 \%)$ desempeñaban algún tipo de labor.

La muestra clínica estuvo conformada por 75 personas, atendidas en instituciones de salud o geriátricas, con diagnóstico de demencia soportado en la historia clínica, quienes fueron seleccionadas mediante un muestreo no probabilístico de sujetos tipo, con el apoyo de los profesionales de psicología y de psiquiatría. La muestra no clínica la constituyeron 138 participantes sin diagnóstico clínico, quienes conocían el objetivo del estudio y voluntariamente decidieron participar. Se excluyeron los participantes con limitaciones visuales o auditivas avanzadas que carecían de los dispositivos para la corrección (gafas y audífonos), y quienes tenían antecedentes de enfermedad psiquiátrica, abuso crónico de sustancias, trastornos neurológicos o presentaban una depresión establecida en el momento de la evaluación, según los resultados del GDS (puntuación igual o superior a 10). Dado que no se contó con el diagnóstico de neurología o neuropsicología sobre el grado en la escala GDS o CDR, se trabajó con datos dicotómicos: 1. Tiene demencia; 2 . No tiene demencia.

\section{Instrumentos}

Encuesta de Datos Sociodemográficos. Diseñada por Avendaño y Avendaño (2009).

Test de cribado para la evaluación de las demencias en adultos mayores (Eurotest/Pesotest). Prueba original elaborada por Carnero y Montoro (2004), denominada Eurotest, adaptada por Avendaño y Avendaño (2009) con el nombre de Pesotest. Su objetivo es determinar si el evaluado presenta un compromiso cognitivo mediante una secuencia de tareas que involucran seguimiento de instrucciones, manipulación de monedas nacionales (pesos) de denominación común $(50,100,200$ y 500), operaciones matemáticas con el dinero y evocación en el largo e inmediato plazo de información específica (Véase apéndice A). Estos autores, con una muestra colombiana de 75 participantes, encontraron un punto de corte de 18, consistencia interna con el Alpha de Cronbach de 0,85 y una estructura factorial de tres factores, los cuales explican el $62,45 \%$ de la varianza y que corroboraron los resultados planteados por el autor.

Mini Mental State Examination (MMSE). Instrumento de cribaje para el diagnóstico del deterioro cognitivo y la demencia. Su versión original fue propuesta por Folstein, Folstein y McHugh (1975). Rosselli et al. (2000) lo adaptaron al contexto colombiano como instrumento de tamizaje para clasificar al grupo de adultos evaluados y comparar los resultados arrojados con cada una de las pruebas. Consta de 19 ítems que evalúan orientación temporal y espacial, recuerdo inmediato, cálculo, evocación, denominación de objetos, repetición, comprensión y seguimiento de órdenes verbales y escritas, escritura y copia de un diagrama. Los resultados arrojaron que el MMSE tenía una sensibilidad de $92.3 \%$ y una especificidad de $53,7 \%$; las puntuaciones tienen una alta correlación con el nivel educativo.

Escala de Depresión Geriátrica de Yesavage (GDS). Fue diseñada por Brink et al. (1982) para evaluar el estado afectivo en el adulto mayor. Se retomó el cuestionario utilizado por Gómez-Angulo, y Campo-Arias (2011) en población colombiana; tiene 15 ítems con respuesta dicotómica (sí o no); presentó consistencia interna de 0.78 , confiabilidad de constructo de 0.87 y estructura bidimensional (desesperanza y estado de ánimo deprimido). 


\section{Procedimiento}

La investigación siguió los lineamientos éticos del Ministerio de Salud (1993) y el Congreso de la República de Colombia (2006). Se desarrolló en cuatro fases:

Fase I: Se tramitó el permiso para la aplicación y el análisis psicométrico del test original denominado Eurotest por su autor, el doctor Cristóbal Carnero; se gestionó la autorización para la aplicación de los instrumentos a las instituciones prestadoras de salud e instituciones geriátricas que atendieran adultos mayores con y sin diagnóstico de demencia, identificadas previamente. Se solicitó la firma del consentimiento informado a los participantes de la muestra no clínica y al familiar o representante de los participantes de la muestra clínica.

Fase II: Se aplicaron los instrumentos con la colaboración de psicólogos, estudiantes de último año de psicología, un fisioterapeuta y una auxiliar de enfermería, previamente instruidos sobre la aplicación.

Fase III: Se registraron los datos en Excel, que luego se exportaron a SPSS (versión 20). Se depuró la base de datos y se realizó el análisis descriptivo de cada una de las variables sociodemográficas. Se obtuvieron los puntajes para las dos muestras en cada una de las pruebas, se estableció la normalidad de las distribuciones y la diferencia entre las medias de los puntajes de las subpruebas y de los totales de cada prueba por muestra.

Fase IV: Se analizaron las propiedades psicométricas del Pesotest. Bajo la teoría clásica de los test (TCT), con el software SPSS, se estableció la consistencia interna de cada una de las partes y de la prueba total del Pesotest, con el Alfa de Cronbach. Se determinó la validez convergente con el coeficiente de correlación producto momento de Pearson entre los puntajes obtenidos en el Pesotest y el MMSE, y la validez de constructo del Pesotest mediante análisis factorial exploratorio. Bajo la teoría de respuesta al ítem (TRI), se estableció la comprobación del ajuste de los ítems con datos empíricos al modelo de Rasch, con el software Winsteps, procedimiento que no se puede realizar con TCT. La TRI no contradice los supuestos de la TCT, por lo cual se consideró pertinente la utilización de los dos modelos.

\section{RESULTADOS}

Inicialmente se estableció la normalidad de las distribuciones a partir del estadístico Kolmogorov-Smirnov, y se encontró que las distribuciones del Pesotest y del MMSE, tanto en muestra clínica como no clínica, eran normales. Se presentan las diferencias de medias en los puntajes obtenidos en el Pesotest y el MMSE; posteriormente se relacionan las propiedades psicométricas del Pesotest: la consistencia interna de cada una de las tres partes y de la prueba total; la validez convergente y la validez de constructo. Finalmente, se presentan los puntajes de sensibilidad y especificidad y el punto de corte para el Pesotest.

Diferencias de medias en los puntajes obtenidos en el Pesotest y el MMSE

En las subpruebas que evalúan cálculo y memoria en el Pesotest y en el MMSE, así como en los totales de cada una de estas pruebas, se encontraron diferencias estadísticamente significativas entre las muestras clínica y no clínica (Véanse tablas 1 y 2). En los componentes evaluados, la desviación estándar de la muestra clínica siempre fue mayor a la de la muestra no clínica. Para establecer el tamaño del efecto con los puntajes del Pesotest y del MMSE se utilizó la $d$ de Cohen, con la cual se obtuvo un puntaje de 1,61 y 1,68 respectivamente, que según Ledesma, Macbeth, \& Cortada de Kohan (2008) son efectos altos.

Tabla 1.

Diferencias de medias por tipo de muestra (clínica y no clínica) en cada una de las partes y en los totales del Pesotest

\begin{tabular}{|c|c|c|c|c|c|c|c|}
\hline Subprueba & $\begin{array}{c}M \\
\text { Muestra clínica }\end{array}$ & $\begin{array}{c}M \\
\text { Muestra no clínica }\end{array}$ & $\begin{array}{c}s \\
\text { Muestra clínica }\end{array}$ & $\begin{array}{c}S \\
\text { Muestra } \\
\text { no clínica }\end{array}$ & $t$ & $p$ & $\begin{array}{c}d \text { de } \\
\text { Cohen }\end{array}$ \\
\hline Total P1 (monedas) & 1,8 & 2,64 & 1,42 & 1,22 & $-5,88$ & 0,00 & \multirow{5}{*}{1,61} \\
\hline Total P2 (billetes) & 1,88 & 4,36 & 2,12 & 1,84 & $-8,43$ & 0,00 & \\
\hline Total Cálculo & 3,8 & 6,75 & 2,48 & 2,45 & $-8,34$ & 0,00 & \\
\hline Total Memoria & 4,27 & 6,83 & 2,59 & 2,13 & $-7,34$ & 0,00 & \\
\hline Total Pesotest & 11,67 & 20,84 & 5,69 & 5,7 & $-11,22$ & 0,00 & \\
\hline
\end{tabular}


Tabla 2.

Diferencias de medias por tipo de muestra (clínica y no clínica) en cada una de las partes y en los totales del MMSE

\begin{tabular}{lcccccc}
\hline Subprueba & $\begin{array}{c}M \\
\text { Muestra clínica }\end{array}$ & $\begin{array}{c}M \\
\text { Muestra no clínica }\end{array}$ & $\begin{array}{c}s \\
\text { Muestra clínica }\end{array}$ & $\begin{array}{c}\text { Muestra } \\
\text { no clínica }\end{array}$ & $\begin{array}{c}t \text { de } \\
\text { Cohen }\end{array}$ \\
\hline Total Cálculo & 1,01 & 2,06 & 1,33 & 1,94 & $-4,55$ & 0,00 \\
Total Memoria & 1,17 & 2,07 & 1,18 & 1,13 & $-5,28$ & 0,00 \\
Total Nominación & 1,91 & 1,97 & 0,33 & 0,24 & $-1,27$ & 0,20 \\
Total MMSE & 18,2 & 26,13 & 4,82 & 3,52 & $-3,18$ & 0,02 \\
\hline
\end{tabular}

Al comparar las medias obtenidas en el Pesotest por las personas que saben y no saben leer y escribir, se encontró un valor $t$ de Student de 1,74 y un $p$ de 0,086 , lo cual indica que no hay diferencias (Véase tabla 3). Con los puntajes obtenidos en el MMSE, también se estableció esta comparación, y se encontró un valor $t$ de Student de 4,21 y un $p=0,00$. Según esta puntuación, las diferencias son estadísticamente significativas (Véase tabla 3).

\section{Consistencia interna}

Con los valores del Alpha de Cronbach de cada una de las pruebas aplicadas, Pesotest 0.86 , MMSE 0.87 y GDS
0.76 , se comprueba que hay consistencia interna en las tres pruebas. Los valores del Alpha de Cronbach de cada una las partes del Pesotest, conocimiento/denominación 0,82 , cálculo 0,73 , recuerdo 0,72 y de la prueba total 0,89 , indican homogeneidad en la prueba y consistencia interna.

\section{Validez convergente}

La validez convergente se estableció mediante el coeficiente de correlación $r$ de Pearson entre las puntuaciones de las subpruebas y el total del Pesotest con las puntuaciones de las subpruebas y el total del MMSE. La tabla 4 muestra que todas las correlaciones fueron estadísticamente significativas, excepto con los totales de denominación.

Tabla 3.

Diferencias de medias en la puntuación obtenida por los participantes en el Pesotest y en el MMSE

\begin{tabular}{lcccccc}
\hline \multicolumn{1}{c}{ Pesotest } & Sabe leer y escribir & $\mathrm{n}$ & $\mathrm{M}$ & $\mathrm{s}$ & $\mathrm{t}$ & $\mathrm{p}$ \\
\hline $\begin{array}{l}\text { Puntuaciones } \\
\text { Totales }\end{array}$ & SI & 179 & 17,94 & 7,349 & & \\
$\begin{array}{l}\text { Pesotest } \\
\text { MMSE }\end{array}$ & NO & 34 & 15,88 & 6,064 & & 0,086 \\
$\begin{array}{l}\text { Puntuaciones } \\
\text { Totales }\end{array}$ & SI & 179 & 22,53 & 6,43 & & \\
MMSE & & & & & 4,21 & 0,000 \\
\hline
\end{tabular}

Tabla 4.

Correlaciones entre los puntajes obtenidos en cada una de las partes y en el total de las pruebas. Muestra total

\begin{tabular}{lcc}
\hline \multicolumn{1}{c}{ Componentes } & $\mathrm{r}$ & $\mathrm{p}$ \\
\hline Total denominación Pesotest Vs Total denominación MMSE & 0.073 & 0.29 \\
Total Cálculo Pesotest Vs Total Cálculo MMSE & $0.584^{* *}$ & 0.00 \\
Total Memoria Pesotest Vs Total Memoria MMSE & $0.483^{* *}$ & 0.00 \\
Total Pesotest Vs Total MMSE & $0.596^{* *}$ & 0.00 \\
\hline
\end{tabular}




\section{Validez de constructo}

La validez de constructo se obtuvo a través del análisis factorial. Se determinó la factibilidad de dicho análisis por medio de la prueba de esfericidad de Bartlett y la prueba Kaiser-Meyer-Olkin (KMO). La primera arrojó un valor de 751,23 con un nivel de significancia $p=0.00$. La segunda dio un valor de 0,845 , indicadores que permiten realizar el análisis.

El análisis factorial arrojó cuatro factores que explican el $61.65 \%$ de la varianza total. Este procedimiento se estimó a partir del método de factores principales. Las cargas factoriales de los componentes se establecieron con rotación Varimax y para el análisis se tuvieron en cuenta las superiores a 0,30.

La tabla 5 muestra los factores y la forma en que se agruparon los ítems de cada parte de la versión del Pesotest. Los ítems 1, 2, 10.1, 10.2, 10.3 y 10.4 conforman el primer factor, que se ha denominado Conocimiento/Denominación-Memoria. Aunque estos ítems también tienen carga en el cuarto factor, Conocimiento/Denominación, Cálculo. El ítem 3 (“¿Cuántas monedas hay aquí?”) comparte su carga entre los factores 2, denominado Cálculo y 4 denominado Conocimiento/Denominación y Cálculo. Los ítems 3, 4, 5 , 6 y 7 conforman el segundo factor, Cálculo. Los ítems 8 y 9 conforman el tercer factor, Memoria y Cálculo.

La tabla 6 presenta los puntajes obtenidos para el punto de corte, sensibilidad, especificidad y Alpha de Cronbach en las cuatro versiones del Eurotest/Pesotest.

\section{Resultados con el análisis Rasch}

Teniendo en cuenta que el modelo exige unidimensionalidad, se realizó el análisis de Rasch por factor y con el total de la prueba. Se usaron dos medidas de ajuste: Outfit o medida sensible al comportamiento inesperado alejado de la media e Infit o medida sensible al comportamiento inesperado cercano a la media (Burga, 2005). Estas medidas tienen un valor esperado de 1.0 y oscilan entre cero e infinito. Valores inferiores a 0,8 indican que los datos no muestran mucha aleatoriedad y valores superiores a 1,3 que los datos presentan demasiada aleatoriedad (González, 2008). Según los resultados, 20 de los 21 ítems se ajustan al modelo de Rasch; el ítem p3 fue el único ítem que no se ajustó al modelo tanto en el análisis por factor como en el análisis del total de la prueba. El error de medida tuvo valores entre 0.12 y 0.27 , la media del error en el total de la prueba fue de 0.15 . Los ítems que mejor identifican el deterioro cognitivo son el ítem denominado moneda 3 (Conocimiento/Denominación de la moneda de \$200) y los ítems p8 (“¿Cuántas monedas le enseñé antes?”) y p9 (“¿Cuánto dinero había en total?”). En general, estos resultados evidencian un alto grado de ajuste de las estimaciones de los ítems (Véase tabla 7). Del total de la prueba solo se presentan la media y la desviación de cada uno de los parámetros analizados.

Tabla 5.

Cargas factoriales de los componentes con rotación superiores a 0,30

\begin{tabular}{|c|c|c|c|c|}
\hline \multirow[b]{3}{*}{ Ítem } & \multicolumn{4}{|c|}{ Matriz de Componentes Principales Rotados } \\
\hline & 1 & 2 & 3 & 4 \\
\hline & $\begin{array}{c}\text { Conocimiento/ } \\
\text { Denominación, } \\
\text { Memoria }\end{array}$ & Cálculo & $\begin{array}{l}\text { Memoria } \\
\text { y cálculo }\end{array}$ & $\begin{array}{c}\text { Conocimiento/ } \\
\text { Denominación, } \\
\text { Cálculo }\end{array}$ \\
\hline subp1 & ,396 & & & 661 \\
\hline subp2 &, 582 & & & ,449 \\
\hline p3 & & 304 & & ,701 \\
\hline $\mathrm{p} 4$ & & ,430 & & \\
\hline $\mathrm{p} 5$ & & ,516 & & \\
\hline p6 & & ,866 & & \\
\hline $\mathrm{p} 7$ & &, 821 & & \\
\hline p8 & & & ,795 & \\
\hline p9 & & & ,637 & \\
\hline p101 &, 743 & & & \\
\hline p102 &, 730 & & & \\
\hline p103 & ,666 & & & \\
\hline p104 & ,742 & & & \\
\hline
\end{tabular}


Tabla 6.

Puntajes obtenidos en las diferentes versiones Eurotest/Pesotest

\begin{tabular}{lcccc}
\hline & $\begin{array}{c}\text { EUROTEST (Carnero, } \\
\text { \& Montoro, 2004) }\end{array}$ & $\begin{array}{c}\text { EUROTEST } \\
(\text { Carnero, 2005) }\end{array}$ & $\begin{array}{c}\text { EUROTEST / PESOTEST } \\
\text { (Avendaño \& Avendaño, 2009) }\end{array}$ & $\begin{array}{c}\text { PESOTEST (Cantor } \\
\& \text { Avendaño, 2015) }\end{array}$ \\
\hline Punto de corte & 23 o menos & $20 / 21$ & 18 & $17 / 18$ \\
Sensibilidad & 0,93 & 0,91 & 0,92 & 0,83 \\
Especificidad & 0,87 & 0,82 & 0,84 & 0,78 \\
Alpha de Cronbach Prueba Total & & & 0.85 & 0.86 \\
\hline
\end{tabular}

Tabla 7.

Estadísticos de ajuste al modelo de Rasch, medida de los ítems en logits y error de medida

\begin{tabular}{|c|c|c|c|c|c|c|c|c|c|}
\hline \multirow{2}{*}{ Factores } & \multirow{2}{*}{$\begin{array}{c}\text { Nombre del } \\
\text { Ítem }\end{array}$} & \multirow{2}{*}{$\begin{array}{l}\text { Orden del } \\
\text { Ítem }\end{array}$} & \multirow{2}{*}{$\begin{array}{c}\text { Total } \\
\mathrm{n}\end{array}$} & \multirow{2}{*}{$\begin{array}{c}\text { Medida } \\
\text { Parámetro }\end{array}$} & \multirow{2}{*}{ SE } & \multicolumn{2}{|c|}{ INFIT } & \multicolumn{2}{|c|}{ OUTFIT } \\
\hline & & & & & & MNSQ & ZSTD & MNSQ & ZSTD \\
\hline \multirow{15}{*}{ Factor 1} & $\mathrm{~mol}$ & 1 & 213 & -0.09 & 0.18 & 1.23 & 2.5 & 1.33 & 2.0 \\
\hline & mo2 & 2 & 213 & -0.77 & 0.19 & 1.22 & 2.0 & 1.32 & 1.4 \\
\hline & mo3 & 3 & 213 & -0.28 & 0.18 & 1.01 & 0.2 & 1.04 & 0.3 \\
\hline & mo4 & 4 & 213 & -0.06 & 0.18 & 1.12 & 1.4 & 1.17 & 1.2 \\
\hline & bille1 & 5 & 213 & -0.18 & 0.18 & 0.94 & -0.7 & 0.76 & -1.6 \\
\hline & bille2 & 6 & 213 & 0.30 & 0.17 & 0.86 & -1.9 & 0.80 & -1.6 \\
\hline & bille3 & 7 & 213 & 0.56 & 0.17 & 0.87 & -1.8 & 0.76 & -2.1 \\
\hline & bille4 & 8 & 213 & 0.67 & 0.17 & 0.86 & -2.0 & 0.83 & -1.5 \\
\hline & bille5 & 9 & 213 & 0.18 & 0.17 & 0.79 & -2.8 & 0.75 & -2.0 \\
\hline & bille6 & 10 & 213 & -0.06 & 0.18 & 0.87 & -1.6 & 0.85 & -1.0 \\
\hline & p101 & 18 & 213 & -1.03 & 0.14 & 0.83 & -1.4 & 0.83 & -0.7 \\
\hline & p102 & 19 & 213 & 0.24 & 0.13 & 1.04 & 0.4 & 1.05 & 0.5 \\
\hline & p103 & 20 & 213 & 0.66 & 0.13 & 1.20 & 2.2 & 1.18 & 1.9 \\
\hline & p104 & 21 & 213 & 0.16 & 0.12 & 1.13 & 1.1 & 1.31 & 1.4 \\
\hline & p3 & 11 & 213 & -2.19 & 0.16 & 1.43 & 2.7 & 3.95 & 4.5 \\
\hline \multirow{4}{*}{ Factor 2} & $\mathrm{p} 4$ & 12 & 213 & -1.42 & 0.13 & 0.81 & -1.5 & 1.60 & 1.4 \\
\hline & p5 & 13 & 213 & 0.13 & 0.12 & 1.06 & 0.6 & 1.11 & 0.6 \\
\hline & p6 & 14 & 213 & 1.62 & 0.13 & 0.80 & -1.6 & 0.46 & -1.6 \\
\hline & p7 & 15 & 213 & 1.86 & 0.14 & 0.67 & -3.0 & 0.46 & -3.0 \\
\hline \multirow{3}{*}{ Factor 3} & $\mathrm{p} 8$ & 16 & 213 & 1.04 & 0.27 & 1.00 & 0.0 & 1,00 & 0.0 \\
\hline & p9 & 17 & 213 & -1.04 & 0.27 & 1.00 & 0.0 & 1,00 & 0.0 \\
\hline & mol & 1 & 213 & 0.00 & 0.18 & 1.14 & 1.6 & 1.17 & 1.3 \\
\hline \multirow{10}{*}{ Factor 4} & mo2 & 2 & 213 & -0.69 & 0.19 & 1.11 & 1.1 & 1.45 & 2.3 \\
\hline & mo3 & 3 & 213 & -0.20 & .0 .18 & 0.95 & -0.6 & 0.95 & -0.3 \\
\hline & mo4 & 4 & 213 & 0.03 & 0.18 & 1.10 & 1.2 & 1.12 & 1.0 \\
\hline & bille1 & 5 & 213 & 1.10 & 0.18 & 0.90 & -1.2 & 0.76 & -1.9 \\
\hline & bille2 & 6 & 213 & 0.41 & 0.17 & 0.79 & -2.9 & 0.71 & -2.8 \\
\hline & bille3 & 7 & 213 & 0.68 & 0.17 & 0.89 & -1.5 & 0.86 & -1.2 \\
\hline & bille4 & 8 & 213 & 0.80 & 0.17 & 0.88 & -1.7 & 0.83 & -1.5 \\
\hline & bille5 & 9 & 213 & 0.28 & 0.18 & 0.79 & -2.8 & 0.73 & -2.5 \\
\hline & bille6 & 10 & 213 & 0.03 & 0.18 & 0.90 & -1.3 & 0.91 & -0.7 \\
\hline & p3 & 11 & 213 & -1.25 & 0.15 & 1.72 & 4.1 & 2.98 & 4.4 \\
\hline \multirow{2}{*}{ Total de la prueba } & $\mathrm{M}$ & & 213 & 0.00 & 0.15 & 1.00 & -0.2 & 1.11 & 0.0 \\
\hline & $\mathrm{s}$ & & 0.1 & 0.93 & 0.03 & 0.18 & 1.6 & 0.55 & 1.8 \\
\hline
\end{tabular}


La medida del parámetro se presenta en logits e indica la dificultad de cada ítem. Los valores oscilaron entre -2.19 para el ítem p3, el más fácil, y 1.86 para el ítem p7, el más difícil. Se encontraron 11 ítems con puntuaciones superiores a la media de 0 logits y 10 por debajo de la dificultad media.

El punto de corte, la sensibilidad y especificidad de la prueba se calcularon contrastando las respuestas de la muestra no clínica $(\mathrm{n}=138)$ con la muestra clínica $(\mathrm{n}=75)$, mediante Curvas de Características Operantes del Receptor (Curvas ROC, por sus siglas en inglés, Receiver Operating Characteristics). Los resultados indicaron un punto de corte de 18 , sensibilidad (83\%) y especificidad (78\%).

Respecto al DIF, se conoce que "un ítem funcionará diferencialmente o presenta DIF, cuando dos grupos comparables de sujetos, es decir, con un nivel idéntico respecto al atributo medido por el test, lo ejecuten de manera distinta" (Hidalgo, Galindo, Inglés, Campoy \& Ortiz, 1999; p.331). Teniendo en cuenta esta definición no se consideró relevante aplicar DIF, puesto que los grupos no son comparables; de entrada se espera mejor desempeño en la muestra no clínica. De hecho, un estudio anterior con el Pesotest, realizado con otra muestra por Avendaño, Avendaño y Cruz (2014), en cuyos objetivos se incluía analizar las diferencias por ítem entre las muestras, utilizó DIF y mostró que, efectivamente, los resultados de las dos muestras por ítem eran diferentes.

\section{DISCUSIÓN}

Esta investigación determinó las propiedades psicométricas del test de cribado de demencias Pesotest en muestras clínica y no clínica de adultos mayores. Las diferencias estadísticamente significativas de las puntuaciones obtenidas en el Pesotest entre las muestras clínica y no clínica, con promedios más altos para la muestra no clínica en cada una de las subpruebas y mayores varianzas para la muestra clínica, indican mayor homogeneidad en las puntuaciones de la muestra no clínica.

Las puntuaciones obtenidas por los participantes alfabetas $\mathrm{y}$ analfabetas no evidenciaron diferencias significativas en el Pesotest, lo cual señala que está libre de sesgo educativo, mientras que las diferencias significativas en el MMSE muestran un sesgo de escolaridad para esta prueba. Esto confirma los hallazgos de Carnero (2005), Avendaño y Avendaño (2009) y Martínez (2012) en cuanto a la utilidad del Pesotest para realizar el cribado, en especial en poblaciones con bajo nivel educativo y altas tasas de analfabetismo. Teniendo en cuenta que el $16 \%$ de esta muestra y alrededor del $20 \%$ de los adultos mayores colombianos carecen de estudio (Profamilia, 2010), se enfatiza en la necesidad de realizar una evaluación libre de sesgo educativo, rápida, confiable y aplicable a analfabetas; aspectos que fueron tenidos en cuenta por González (2012) para describir el desempeño neuropsicológico con el Pesotest en los procesos de denominación, cálculo, memoria de trabajo y memoria episódica, como funciones cognitivas esenciales requeridas para el manejo del dinero por parte de un grupo de adultos mayores analfabetas sin escolaridad e institucionalizados.

Respecto a la consistencia interna, los valores del Alfa de Cronbach de cada uno de los componentes propuestos en la versión original indican homogeneidad en la prueba de cada una de las partes del Pesotest, aspecto que corrobora los hallazgos de Avendaño y Avendaño (2009). Se comprobó que la prueba tiene validez convergente; por lo tanto, el Pesotest puede ser utilizado en lugar del MMSE, en razón de que también permite realizar el cribado.

Sobre la validez de constructo, Carnero (2005) afirma que este test de cribado agrupa sus ítems en tres factores: 1) Conocimiento-Denominación, 2) Cálculo y 3 ) Memoria. Los hallazgos de Avendaño y Avendaño (2009) coinciden con este número de factores. Aunque los resultados del presente estudio mostraron que el Pesotest se distribuyó en cuatro factores, es importante clarificar que no se propone un dominio adicional a los reportados por los autores del Eurotest, sino que se plantea una combinación de los propuestos inicialmente por ellos.

En este punto es pertinente señalar que para establecer el diagnóstico de demencia se exige el compromiso de varios dominios cognitivos (memoria y al menos otro más). El análisis de los componentes del Pesotest evidencia que incluye la evaluación de memoria y cálculo. En esta investigación, dos de los cuatro factores en el Pesotest incluyen la evaluación del dominio memoria (Factor 1) Conocimiento/Denominación-Memoria; Factor 3) Memoria y cálculo) y los otros dos: Factor 2) Cálculo y Factor 4) Conocimiento/Denominación-Cálculo) permiten evaluar el dominio Cálculo, aspecto que favorece al Pesotest, pues se ha encontrado que este último dominio es uno de los mejores predictores del rendimiento cognoscitivo, tanto en personas normales como en pacientes con la enfermedad de Alzheimer (Rosselli et al., 2000). Según Heun et al. (1998, como se citó en Carnero, 2005), se ha comprobado empíricamente que los instrumentos que evalúan varios dominios cognitivos tienen también una mayor validez en la detección precoz de deterioro cognitivo y de demencia.

El análisis con el modelo de Rasch evidenció que 20 de los 21 ítems se ajustan al modelo, y que el ítem con mayor dificultad fue el p7, que evalúa cálculo. Se corrobora lo establecido por Ferreira, Campagna, Colmenares y Suárez (2008), quienes señalan que los principales indicadores de evolución a demencia son fallas en las funciones ejecutivas, aspecto que se debe tener en cuenta para evaluar la demencia. El ítem p3 no se ajustó al modelo, tanto en el análisis de 
Rasch por factor como en el análisis del total de la prueba, lo cual corrobora los resultados encontrados con la TCT, puesto que según el análisis factorial, este ítem compartió carga en dos factores: el 2 y el 4. En el factor 2 tuvo un Outfit de 3.95, y en el factor 4 tuvo un Infit de 1.72 y un Outfit de 2.98, puntajes que revelan desajuste al modelo.

El punto de corte sugerido por Carnero (2005) para la detección de la demencia con el Eurotest es de 20 puntos con una sensibilidad de 0.91 y una especificidad de 0.82 . El punto de corte sugerido por Avendaño y Avendaño (2009) para la detección de la demencia con el Pesotest es de 18 puntos con una sensibilidad de 0,92 y una especificidad de 0,84 . Basados en los resultados del presente estudio, el punto de corte sugerido para el Pesotest es también de 18 puntos con una sensibilidad de 0.83 y una especificidad de 0.78 . Para garantizar mayor confianza en los resultados obtenidos en el anterior estudio realizado por Avendaño y Avendaño (2009), se amplió el tamaño de las muestras; todos los participantes fueron personas mayores de 65 años (edad a partir de la cual se presenta mayor prevalencia de demencias), y se controló que ningún participante tuviera depresión en el momento de la aplicación del test. Aunque la especificidad encontrada en la presente investigación fue menor a la reportada en los anteriores estudios, es probable que varios de los participantes de la muestra no clínica, cuyas edades oscilaron entre 65 y 94 años, estén comenzando un período demencial no diagnosticado. Los resultados permiten confirmar la capacidad predictiva del Pesotest como prueba de cribado para el diagnóstico temprano de las demencias, así como del deterioro cognitivo.

Una de las limitaciones para el desarrollo de la investigación fue la dificultad para realizar una conformación más específica de la muestra clínica. Esta limitación se asocia con los siguientes hechos: 1) El diagnóstico de demencia en la mayoría de instituciones geriátricas es emitido por profesionales de psiquiatría y psicología, por lo que en las historias clínicas no se registran los datos correspondientes al grado en la escala GDS o CDR establecido por neurología o neuropsicología. 2) No se cuenta con un concepto basado en la evaluación de profesionales especializados de neurología o neuropsicología sobre la ausencia de demencia o de deterioro cognitivo leve en la muestra no clínica. 3) Potenciales participantes fueron excluidos por el estado avanzado de sus limitaciones sensoriales y motoras. 4) Algunas entidades especializadas en demencias, que tienen el servicio de neurología y neuropsicología, previamente habían facilitado a varios investigadores el acceso para la valoración de dicha población o se encontraban participando en ese momento en procesos investigativos que requerían. 5) Existe restricción por parte de las entidades para permitir el acceso a proce- sos investigativos, debido a políticas institucionales o a la negativa de los familiares a participar en ellos.

Para corregir la limitación mencionada, se ratifica la sugerencia que realizaron Avendaño y Avendaño (2009) sobre la importancia de dar continuidad a investigaciones relacionadas con el análisis psicométrico del Pesotest, especialmente en instituciones que cuenten con el apoyo de profesional especializado (neurología o neuropsicología). Este apoyo permitirá clasificar y agrupar a los participantes con base en el grado de demencia establecido por los puntajes de la GDS o en los CDR; incluir el deterioro cognoscitivo leve, y dar una mayor profundidad a las consideraciones de la utilidad diagnóstica del test, para que tanto el área de psicometría como las áreas académicas y de la salud (medicina, neurología, neuropsicología y psicología) se beneficien de los hallazgos obtenidos para su utilización.

Para el desarrollo de futuras investigaciones, se sugiere garantizar que los adultos mayores con déficit auditivo y visual moderado utilicen los dispositivos para la corrección (gafas y audífonos) en el momento de la aplicación, y excluir a quienes presentan déficit sensorial y motor avanzado.

Además de los resultados favorables del análisis psicométrico del Pesotest, este cumple con las características de aplicabilidad relacionadas por Carnero (2005): facilidad de aplicación, corroborada por los profesionales que colaboraron en este estudio; simplicidad y economía, al involucrar únicamente el formato del Pesotest y 11 monedas de fácil disposición; aceptabilidad, dado que evita sesgos por nivel educativo o analfabetismo; equidad, ya que no discrimina entre los participantes; adaptabilidad respecto a las condiciones culturales y étnicas de los participantes, y flexibilidad, que conlleva la capacidad del instrumento para adaptarse al objetivo exacto que se persigue (detectar frente a confirmar) y a la dificultad de cada caso.

Con base en los resultados de las propiedades psicométricas y las características de aplicabilidad del Pesotest, se recomienda su utilización en los servicios de atención primaria como instrumento de cribado con utilidad predictiva en el diagnóstico de demencias. Cualquier profesional que sospeche de la existencia de un deterioro cognitivo o demencia, al aplicar el Pesotest tendrá la posibilidad de efectuar la remisión de los consultantes a los profesionales especializados para completar el estudio.

\section{REFERENCIAS}

Arango, J., Fernández, S., \& Ardila, A. (2003). Las demencias. Aspectos clínicos, neuropsicológicos y tratamiento. México: Manual Moderno.

Avendaño, B., \& Avendaño, X. (2009). Análisis psicométrico del Test de Cribaje para la evaluación de las demencias 
(Eurotest/Pesotest) en una muestra de la ciudad de Bogotá. Tesis de grado: Pontificia Universidad Javeriana.

Avendaño, B.L, \& Avendaño, X.A., \& Cruz, W. (2014). Adaptación al contexto colombiano del test de cribado para la evaluación de la demencia (Eurotest). Revista Avances en Medición, 9, 58-72. http://blog.platform.issuu.com/gestiondeproyectos/ docs/revista_avances_en_medici_n_no._9.

Brink, T.L., Yesavage, J.A., Lum, O., Heersema, P.H., Adey, M., \& Rose, T.L. (1982). Screening tests for geriatric depression. Clinical Gerontologist, 1, 37-44.

Burga, A. (2005). Evaluación del rendimiento académico. Introducción a la TRI. Ministerio de Educación de la República del Perú.

Carnero, C., \& Montoro, M. (2004). Evaluación preliminar de un nuevo test de cribado de demencia (Eurotest). Revista de Neurología, 38(3), 201-209.

Carnero, C. (2005). El Eurotest: test europeo de detección del deterioro cognitivo. Universidad de Granada: Memoria para optar al grado de Doctor en Medicina y Cirugía. Recuperado de https://www.researchgate.net/publication/46589269_El_EUROTEST_test_europeo_de_deteccion_de_deterioro_cognitivo

Congreso de la República de Colombia. (2006). Ley 1090 del 6 de septiembre de 2006. Bogotá: Diario Oficial No. 46.383. Código de Ética del Psicólogo en Colombia. Recuperado de http://www.urosario.edu.co/observatorio-legislativo/Leyessancionadas1/Documentos-2006/2006/ley_1090de06_c/

Díaz, R., Ruano, M.I., Chacón, J.A., \& Vera, A. (2006). Perfil Neuroepidemiológico en la zona centro del departamento de Caldas (Colombia), años 2004-2005. Revista de Neurología, 43, 646-652.

Ferreira, A., Campagna, I., Colmenares, M.F., \& Suárez, J. (2008). Indicadores neuropsicológicos de evolución a demencia tipo Alzheimer en pacientes diagnosticados con deterioro cognitivo leve. Revista de Psicología-Escuela de Psicología, Universidad Central de Venezuela, 27, 2.

Folstein, M., Folstein, S., \& McHugh, P. (1975). Mini-Mental State. A practical method for grading the cognitive state of patients for the clinician. Journal of Psychiatric Research, 12, 189-198.

Gómez-Angulo, C., \& Campo-Arias, A. (2011). Escala de Yesavage para Depresión Geriátrica (GDS-15 y GDS-5): estudio de la consistencia interna y estructura factorial. Universitas Psychologica, 10(3), 735-743.

González, J. (2008). El Análisis de Reactivos con el Modelo Rasch. Manual Técnico A. Serie: Medición y Metodología. Instituto Nacional para la Evaluación de la Educación. Universidad de Sonora. México. D. F.

González, M. (2012). Desempeño neuropsicológico según la prueba Pesotest de adultos mayores analfabetas no escolarizados e institucionalizados-Bogotá 2012. Tesis de grado: Universidad de San Buenaventura Sede Bogotá, Maestría en Neuropsicología Clínica.
Gooding, M.P., Amaya, E., Parra, M., \& Ríos, A.M. (2006). Prevalencia de las demencias en el municipio de Neiva 20032005. Acta Neurológica Colombiana, 22, 243-248.

Hidalgo, M. D., Galindo, F., Inglés, C. J., Campoy, G. \& Ortiz, B. (1999). Estudio del funcionamiento diferencial de los ítems en una escala de habilidades sociales para adolescentes. Anales de Psicología, 15(2), 331-343.

Kerlinger, F., \& Lee, H. (2002). Investigación del comportamiento: métodos de investigación en ciencias sociales. México: Mc Graw Hill.

Ledesma, R., Macbeth, G., \& Cortada de Kohan, N. (2008). Tamaño del efecto: revisión teórica y aplicaciones con el sistema estadístico ViSta. Revista Latinoamericana de Psicología, 40(3), 425-439.

Martínez, M. A. (2012). Perfil neuropsicológico en adultos mayores colombianos sanos analfabetas y con bajo nivel educativo. Universidad Nacional: Trabajo de investigación presentado como requisito parcial para optar al título de Magister en Neurociencias.

McIntire, S., \& Miller, L. (2000). Foundations of psychological testing. Boston: McGraw Hill.

Meulen, E. F., Schmand, B., Van Campen, J. P., De Koning, S. J., Ponds, R. W., Scheltens, P., \& Verhey, F. R. (2004). The seven minutes screen: A neurocognitive screening test highly sensitive to various types of dementia. Journal of Neurology, Neurosurgery and Psychiatry, 75(5), 700-705.

Ministerio de Salud (1993). Resolución 8430 por la cual se dictan normas éticas y legales de la investigación clínica aplicada. Recuperado de https://www.minsalud.gov.co/Normatividad_Nuevo/RESOLUCION\%208430\%20DE\%201993.pdf

Montero, I., \& León, O.G. (2007). A guide for naming research studies in Psychology. International Journal of Clinical and Health Psychology, 7 (3), 847-862. Recuperado de http:// www.aepc.es/ijchp/GNEIP07_es.pdf

Nunnally, J., \& Bernstein, I. (1995). Teoría psicométrica. México: Mc Graw Hill.

Ostroskyj-Solis, F., López - Arango, G., \& Ardila, A. (1999). Influencias de la edad y la escolaridad en el examen breve del estado mental (Mini-Mental State Examination) en una población hispano-hablante. Salud Mental, 22(3), 20 - 26.

Peña-Casanova, J., Gramunt, N., \& Gich, J. (2004). Test neuropsicológicos. Fundamentos para una neuropsicología clínica basada en evidencias. Barcelona: Masson, S.A.

Pradilla, G., \& Vesga, B. (2002). Estudio Neuroepidemiológico en Piedecuesta (Santander). Acta Médica Colombiana, 27, 407-420.

Pradilla, G., Vesga, B.E., \& Bautista, L.E. (2000). Neuroepidemiología en Piedecuesta, población semiurbana de Santander. Acta Médica Colombiana, 25, 286.

Pradilla. G., Rosselli, D., \& Bautista, L. (1998). Estudio Neuroepidemiológico Nacional. Acta Médica Colombiana, 23, 258. 
Profamilia. (2010). Encuesta Nacional de Demografia y Salud 2010. Recuperado de http://www.profamilia.org.co/encuestas/ Profamilia/Profamilia/index.php?option=com_content\&view $=$ article \&id $=136 \&$ Itemid $=154$

Rincón, D.L. (2013). ¿Qué está haciendo Colombia por las personas mayores? Fundación Agencia de Comunicaciones Periodismo Aliado de la Niñez, el Desarrollo Social y la Inves- tigación - PANDI. Recuperado de http://www.agenciapandi. org/que-esta-haciendo-colombia-por-las-personas-mayores/ Rosselli, D., Ardila, A., Pradilla, G., Morillo, L., Bautista, L., Rey, O., Camacho, M., \& GENECO. (2000). El examen mental abreviado, Mini-Mental State Examination, como prueba de selección para el diagnóstico de demencia: estudio poblacional colombiano. Revista de Neurología, 30(5), 428-432. 


\section{APÉNDICE A}

\section{PESOTEST}

(Elaborado por Carnero, \& Montoro, 2004; adaptado por Avendaño, \& Avendaño, 2009).

\section{Primera Parte (Conocimiento/Denominación)}

1. "Por favor dígame las denominaciones o valores de las monedas que hay en la actualidad; Tenga en cuenta que le pregunto monedas y no billetes" (Máximo 1 minuto)

Monedas: $\square 50 \square 100 \square 200 \square 500$

$\square$ Otras (especificar): Total correctas: Intrusiones:

* Se le recuerda que aunque ya no circulan, existieron monedas de 10, 20 y 1.000 pesos.

2. "Por favor dígame las denominaciones o valores de los billetes que hay en la actualidad?”. (Máximo un minuto)

Billetes: $\square 1000 \square 2000 \square 5000 \square 10.000 \square 20.000 \square 50.000$

$\square$ Otros (especificar): Total correctas: Intrusiones:

\section{Segunda Parte (Cálculo)}

Coloque delante del sujeto las monedas ( 3 de 500 pesos, 2 de 200 pesos, 4 de 100 pesos y 2 de 50 pesos) y pídale de forma consecutiva las siguientes tareas. En caso de que la contestación sea errónea, hágaselo saber y dele un único nuevo intento. Algunos ítems tienen varias soluciones, todas ellas son válidas. Conceda un máximo de un minuto por cada ítem y pase al siguiente si no ha contestado correctamente en este tiempo o ha agotado los dos intentos.

\begin{tabular}{|l|l|}
\hline $\begin{array}{l}\text { 3. “CCuántas monedas hay aquí?” (11) } \\
\square \text { Correcto } \square \text { Correcto segundo intento } \square \text { Incorrecto }\end{array}$ & \\
\hline $\begin{array}{l}\text { 4. “Cámbieme esta moneda (500) por otras que sumen la misma cantidad de dinero” (retirar dos monedas de } 500 \text { pesos y sólo } \\
\text { se le deja una) } \\
\square \text { Correcto } \square \text { Correcto segundo intento } \square \text { Incorrecto }\end{array}$ & $\begin{array}{l}\text { 5. “CCuánto dinero hay aquí en total?” (\$2.400 pesos) (de nuevo todas las monedas) } \\
\square \text { Correcto } \square \text { Correcto segundo intento } \square \text { Incorrecto }\end{array}$ \\
\hline $\begin{array}{l}\text { 6. “Por favor, reparta estas monedas en dos montones que tengan la misma cantidad de dinero” }(\$ 1200) \\
\square \text { Correcto } \square \text { Correcto segundo intento } \square \text { Incorrecto }\end{array}$ & $\begin{array}{l}\text { 7. “Por favor, reparta estas monedas en tres montones que tengan la misma cantidad de dinero” }(\$ 800 \text { pesos) } \\
\square \text { Correcto } \square \text { Correcto segundo intento } \square \text { Incorrecto }\end{array}$ \\
\hline
\end{tabular}

\section{Tarea de distracción: Fluencia Verbal Semántica}

"Quiero que me diga todos los nombres de animales que se le ocurran, ya sean de la tierra, del mar o del aire, del campo o de la casa, jtodos los que se le ocurran!"

\section{Tercera Parte (Recuerdo)}

"Para finalizar, quiero que haga un último esfuerzo y trate de recordar":

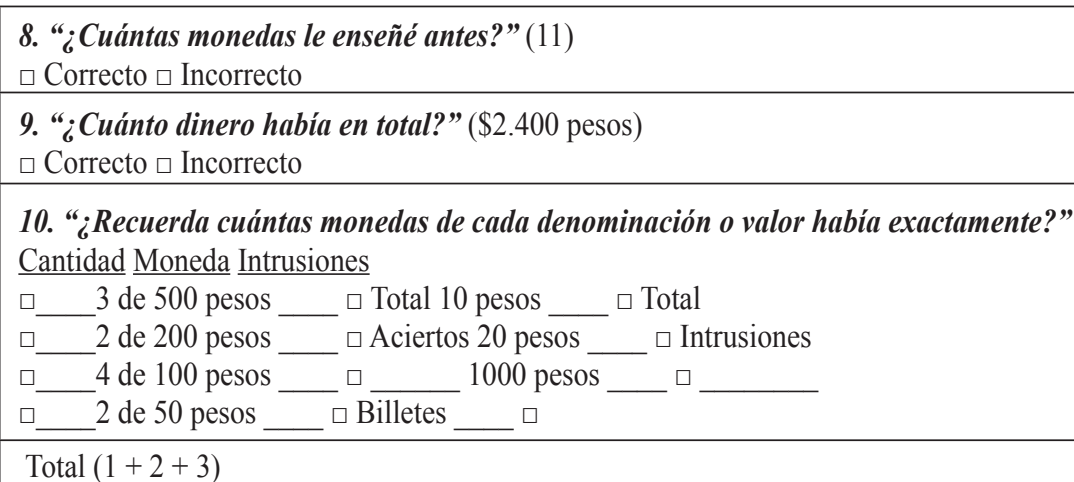

